\title{
New Paradigms for the Peroxy Acid Epoxidation of CC Double Bonds: The Role of the Peroxy Acid s-Trans Conformer and of the 1,2-H Transfer in the Epoxidation of Cyclic Allylic Alcohols.
}

Mauro Freccero, ${ }^{a}$ Remo Gandolfi, ${ }^{a^{*}}$ Mirko Sarzi-Amade ${ }^{a}$ and Augusto Rastelli ${ }^{b}$.
a Dipartimento di Chimica Organica, Università di Pavia, V.le Taramelli 10, 27100 Pavia Italy.
${ }^{\mathrm{b}}$ Dipartimento di Chimica, Università di Modena, Via Campi 183, 41100 Modena, Italy.
remo.gandolfi@unipv.it

\section{Supporting Information Available}

Table of Contents
Energy and cartesian coordinates of reactants
S2-S4
Energy, imaginary frequency and cartesian coordinates of TSs
IRC data
S11-S13
Enthalpy, entropy and free energy of reactants
S14-S15
TS geometries (Table S1 and Table S2).
S16-S17
TS activation parameters (Table S3)
S18 

phase and dichloromethane solution.

s-cis HCOOOH (gas phase)

$\mathrm{E}=-264.972914$

$\begin{array}{rrrr}\mathrm{C} & -0.608553 & -0.000339 & -0.657630 \\ \mathrm{O} & -0.608220 & -0.000028 & 0.684983 \\ \mathrm{O} & 0.729413 & 0.000132 & 1.220427 \\ \mathrm{H} & 1.269597 & -0.000694 & 0.400303 \\ \mathrm{O} & 0.382775 & 0.000255 & -1.337176 \\ \mathrm{H} & -1.650016 & -0.000156 & -1.000393\end{array}$

s-cis $\mathrm{HCOOOH}\left(\mathrm{CH}_{2} \mathrm{Cl}_{2}\right)$

$E=-264.977279$ and (with non electrostatic terms) - 264.976919

$\begin{array}{rrrr}\mathrm{C} & -0.603314 & 0.000904 & -0.668002 \\ \mathrm{O} & -0.612999 & 0.001063 & 0.676687 \\ \mathrm{O} & 0.715589 & -0.001017 & 1.235530 \\ \mathrm{H} & 1.283918 & -0.000624 & 0.432861 \\ \mathrm{O} & 0.394805 & -0.001053 & -1.338166 \\ \mathrm{H} & -1.643201 & 0.003260 & -1.017253\end{array}$

2-cyclobutenol (conformer with $\mathrm{O}-\mathrm{H}$ inside) (gas phase) $\mathrm{E}=-231.260864$

$\begin{array}{lrrr}\mathrm{C} & -0.454282 & -0.217252 & -1.066246 \\ \mathrm{C} & -0.447536 & -0.233564 & 0.454696 \\ \mathrm{C} & 1.126211 & -0.257689 & 0.337277 \\ \mathrm{C} & 0.879021 & -0.303191 & -1.161843 \\ \mathrm{H} & -0.854064 & -1.146195 & 0.896178 \\ \mathrm{H} & 1.633409 & -1.119264 & 0.783458 \\ \mathrm{H} & -1.261637 & -0.197094 & -1.789621 \\ \mathrm{H} & 1.554666 & -0.415801 & -2.002215 \\ \mathrm{O} & -1.060278 & 0.828890 & 1.159800 \\ \mathrm{H} & 1.596631 & 0.657243 & 0.707714 \\ \mathrm{H} & -0.807274 & 1.660161 & 0.742787\end{array}$

2-cyclobutenol (conformer with $\mathrm{O}-\mathrm{H}$ inside) $\left(\mathrm{CH}_{2} \mathrm{Cl}_{2}\right)$

$E=-231.265145$ and (with non electrostatic terms) -231.266122

$\begin{array}{lrrr}\mathrm{C} & -0.444060 & -0.234602 & -1.068336 \\ \mathrm{C} & -0.445832 & -0.238911 & 0.452257 \\ \mathrm{C} & 1.127695 & -0.232361 & 0.345727 \\ \mathrm{C} & 0.891281 & -0.301377 & -1.154508 \\ \mathrm{H} & -0.839009 & -1.157841 & 0.898727 \\ \mathrm{H} & 1.648959 & -1.078162 & 0.806114 \\ \mathrm{H} & -1.246992 & -0.235073 & -1.797460 \\ \mathrm{H} & 1.575042 & -0.413951 & -1.988682 \\ \mathrm{O} & -1.083917 & 0.821366 & 1.147595 \\ \mathrm{H} & 1.576913 & 0.698312 & 0.703932 \\ \mathrm{H} & -0.818083 & 1.659293 & 0.745765\end{array}$

2-cyclopentenol (conformer with O-H inside) (gas phase) $\mathrm{E}=-270.625121$.
C
C
C
C
C
H
0.007465
0.067362
0.028803
0.047221
1. 362960
0.002785
$-0.023270$
1.947758
1.392921
0.083899
$-0.532268$
2. 291685
0.703202
$-0.239081$
0.377621
0.702610 


$\begin{array}{rrrr}\mathrm{H} & 1.632986 & 0.896689 & 2.495106 \\ \mathrm{H} & 1.490907 & -0.839187 & 2.670766 \\ \mathrm{H} & -0.867565 & 0.135938 & -0.606989 \\ \mathrm{H} & -0.888049 & 0.090340 & 1.980474 \\ \mathrm{H} & 2.611790 & -1.283001 & 0.655626 \\ \mathrm{H} & 1.558821 & -0.642921 & -1.333448 \\ \mathrm{O} & 1.713900 & 1.343137 & -1.158109 \\ \mathrm{H} & 1.415643 & 2.048234 & -0.572370\end{array}$

2-cyclopentenol (conformer with $\mathrm{O}-\mathrm{H}$ inside) $\left(\mathrm{CH}_{2} \mathrm{Cl}_{2}\right)$ $E=-270.629216$ and (with non electrostatic terms) -270.631369

$\begin{array}{rrrr}\text { C } & -1.171075 & -0.228947 & -0.346772 \\ \mathrm{C} & -1.169193 & -0.234311 & 0.987688 \\ \mathrm{C} & 0.224486 & -0.235075 & 1.566759 \\ \mathrm{C} & 0.226620 & -0.165241 & -0.915667 \\ \mathrm{C} & 1.129141 & -0.417980 & 0.320942 \\ \mathrm{H} & 1.991741 & 0.251605 & 0.311195 \\ \mathrm{H} & 0.421986 & 0.699954 & 2.105246 \\ \mathrm{H} & 0.363120 & -1.040134 & 2.295248 \\ \mathrm{H} & -2.051849 & -0.207385 & -0.978670 \\ \mathrm{H} & -2.058461 & -0.222750 & 1.609283 \\ \mathrm{H} & 1.510824 & -1.441799 & 0.286302 \\ \mathrm{H} & 0.401654 & -0.905402 & -1.700610 \\ \mathrm{O} & 0.473370 & 1.093743 & -1.577429 \\ \mathrm{H} & 0.194147 & 1.805298 & -0.986258\end{array}$

2-cyclohexenol (pseudoequatorial conformer with O-H inside) (gas phase) $\mathrm{E}=-309.955675$

$\begin{array}{rrrr}\mathrm{C} & -1.091883 & 0.219960 & -1.232939 \\ \mathrm{C} & -1.081703 & 0.233456 & 1.291601 \\ \mathrm{C} & 1.092099 & 0.243199 & 0.003908 \\ \mathrm{C} & 0.316182 & -0.384065 & 1.167231 \\ \mathrm{C} & 0.237316 & 0.346610 & -1.236217 \\ \mathrm{C} & -1.905333 & -0.003695 & 0.016412 \\ \mathrm{H} & 0.222077 & -1.462203 & 0.980390 \\ \mathrm{H} & 0.887731 & -0.269623 & 2.092008 \\ \mathrm{H} & 1.433369 & 1.244294 & 0.292177 \\ \mathrm{H} & -1.603283 & -0.174928 & 2.162059 \\ \mathrm{H} & -0.985277 & 1.312347 & 1.462782 \\ \mathrm{H} & -2.302333 & -1.028811 & 0.007114 \\ \mathrm{H} & -2.785110 & 0.649682 & 0.002615 \\ \mathrm{H} & -1.634938 & 0.282825 & -2.172825 \\ \mathrm{H} & 0.772644 & 0.532090 & -2.163666 \\ \mathrm{O} & 2.313572 & -0.459736 & -0.267012 \\ \mathrm{H} & 2.086471 & -1.340578 & -0.586538\end{array}$

2-cyclohexenol (conformer with $\mathrm{O}-\mathrm{H}$ inside) $\left(\mathrm{CH}_{2} \mathrm{Cl}_{2}\right)$ $E=-309.959651$ and (with non electrostatic terms) - 309.962477

$\begin{array}{rrrr}\mathrm{C} & -1.093000 & 0.222611 & -1.232123 \\ \mathrm{C} & -1.081346 & 0.232647 & 1.291986 \\ \mathrm{C} & 1.091445 & 0.245046 & 0.003674 \\ \mathrm{C} & 0.317271 & -0.383463 & 1.166843 \\ \mathrm{C} & 0.236536 & 0.349108 & -1.236260 \\ \mathrm{C} & -1.905131 & -0.004518 & 0.017133 \\ \mathrm{H} & 0.224733 & -1.461618 & 0.979964 \\ \mathrm{H} & 0.887111 & -0.266085 & 2.092707 \\ \mathrm{H} & 1.434660 & & 1.246219\end{array}$




$\begin{array}{rrrr}\mathrm{H} & -1.601481 & -0.178666 & 2.161819 \\ \mathrm{H} & -0.986134 & 1.311417 & 1.464931 \\ \mathrm{H} & -2.299064 & -1.030737 & 0.006000 \\ \mathrm{H} & -2.786640 & 0.646481 & 0.004319 \\ \mathrm{H} & -1.636738 & 0.287100 & -2.171789 \\ \mathrm{H} & 0.770085 & 0.534783 & -2.165142 \\ \mathrm{O} & 2.314252 & -0.463120 & -0.270191 \\ \mathrm{H} & 2.084811 & -1.352514 & -0.571293\end{array}$

\section{Energy (hartree), imaginary frequency $\left[v_{i}\left(\mathrm{~cm}^{-1}\right)\right]$ and cartesian coordinates of TSs, at the B3LYP/6-311+G(d,p) level, in gas phase and dichloromethane solution}

TS 2a (gas phase)

$\mathrm{E}=-496.217892 ; v_{\mathrm{i}}=-369.7 \mathrm{~cm}^{-1}$

$\begin{array}{lrrr}\mathrm{C} & -1.594314 & -0.044300 & -0.411729 \\ \mathrm{C} & -1.588314 & 1.482700 & -0.397729 \\ \mathrm{C} & -1.565314 & 1.359700 & 1.181271 \\ \mathrm{C} & -1.637314 & -0.133300 & 0.960271 \\ \mathrm{O} & 2.213686 & -0.396300 & 0.040271 \\ \mathrm{O} & 0.369686 & -0.275300 & 0.155271 \\ \mathrm{C} & 2.423686 & -1.651300 & -0.162729 \\ \mathrm{O} & 1.560686 & -2.521300 & -0.252729 \\ \mathrm{H} & -2.528314 & 1.906700 & -0.760729 \\ \mathrm{H} & -2.421314 & 1.799700 & 1.700271 \\ \mathrm{H} & -1.695314 & -0.771300 & -1.207729 \\ \mathrm{H} & -1.769314 & -0.969300 & 1.635271 \\ \mathrm{H} & 0.374686 & -1.261300 & 0.005271 \\ \mathrm{H} & 3.498686 & -1.887300 & -0.252729 \\ \mathrm{O} & -0.558314 & 2.156700 & -1.055729 \\ \mathrm{H} & -0.641314 & 1.708700 & 1.644271 \\ \mathrm{H} & 0.265686 & 1.682700 & -0.876729\end{array}$

TS $2 \mathbf{a}\left(\mathrm{CH}_{2} \mathrm{Cl}_{2}\right)$

$E=-496.226357$ and (with non electrostatic terms) $-496.225500 ; v_{i}=-392.7 \mathrm{~cm}^{-1}$

$\begin{array}{rrrr}\mathrm{C} & -1.640071 & -0.055814 & -0.420286 \\ \mathrm{C} & -1.631071 & 1.469186 & -0.417286 \\ \mathrm{C} & -1.617071 & 1.359186 & 1.159714 \\ \mathrm{C} & -1.684071 & -0.134814 & 0.947714 \\ \mathrm{O} & 2.209929 & -0.387814 & 0.156714 \\ \mathrm{O} & 0.396929 & -0.288814 & 0.192714 \\ \mathrm{C} & 2.487929 & -1.614814 & -0.142286 \\ \mathrm{O} & 1.674929 & -2.507814 & -0.353286 \\ \mathrm{H} & -2.564071 & 1.900186 & -0.796286 \\ \mathrm{H} & -2.481071 & 1.797186 & 1.667714 \\ \mathrm{H} & -1.731071 & -0.788814 & -1.213286 \\ \mathrm{H} & -1.823071 & -0.965814 & 1.629714 \\ \mathrm{H} & 0.369929 & -1.250814 & -0.034286 \\ \mathrm{H} & 3.577929 & -1.787814 & -0.190286 \\ \mathrm{O} & -0.579071 & 2.133186 & -1.070286 \\ \mathrm{H} & -0.699071 & 1.716186 & 1.630714 \\ \mathrm{H} & 0.234929 & 1.652186 & -0.866286\end{array}$


TS 2b (gas phase)

$E=-535.583875 . v_{i}=-357.4 \mathrm{~cm}^{-1}$

$\begin{array}{lrrr}\mathrm{C} & 1.489282 & -0.330603 & -0.244000 \\ \mathrm{C} & 1.476282 & 1.035397 & -0.254000 \\ \mathrm{C} & 1.454282 & 1.601397 & 1.136000 \\ \mathrm{C} & 1.466282 & -0.877603 & 1.166000 \\ \mathrm{C} & 1.158282 & 0.375397 & 2.033000 \\ \mathrm{H} & 2.454282 & -1.289603 & 1.406000 \\ \mathrm{H} & 1.730282 & 0.381397 & 2.961000 \\ \mathrm{H} & 2.437282 & 2.039397 & 1.352000 \\ \mathrm{H} & 0.724282 & 2.407397 & 1.241000 \\ \mathrm{H} & 1.624282 & -0.967603 & -1.110000 \\ \mathrm{H} & 1.562282 & 1.645397 & -1.145000 \\ \mathrm{C} & -2.605718 & -0.009603 & -1.742000 \\ \mathrm{O} & -1.773718 & 0.091397 & -2.639000 \\ \mathrm{O} & -2.361718 & -0.015603 & -0.476000 \\ \mathrm{O} & -0.527718 & 0.166397 & -0.376000 \\ \mathrm{H} & -0.543718 & 0.178397 & -1.371000 \\ \mathrm{H} & -3.684718 & -0.107603 & -1.959000 \\ \mathrm{H} & 0.099282 & 0.353397 & 2.295000 \\ \mathrm{O} & 0.569282 & -1.958603 & 1.338000 \\ \mathrm{H} & -0.284718 & -1.675603 & 0.984000\end{array}$

TS 2b $\left.\left(\mathrm{CH}_{2} \mathrm{Cl}_{2}\right)\right)$

$E=-535.592245$ and (with non electrostatic terms) $-535.592106 ; v_{i}=-384.4 \mathrm{~cm}^{-1}$

$\begin{array}{lrrr}\mathrm{C} & 1.530167 & -0.330897 & -0.233026 \\ \mathrm{C} & 1.518167 & 1.032103 & -0.244026 \\ \mathrm{C} & 1.489167 & 1.601103 & 1.142974 \\ \mathrm{C} & 1.494167 & -0.875897 & 1.175974 \\ \mathrm{C} & 1.191167 & 0.377103 & 2.042974 \\ \mathrm{H} & 2.474167 & -1.301897 & 1.422974 \\ \mathrm{H} & 1.768167 & 0.384103 & 2.967974 \\ \mathrm{H} & 2.472167 & 2.039103 & 1.361974 \\ \mathrm{H} & 0.760167 & 2.409103 & 1.244974 \\ \mathrm{H} & 1.666167 & -0.966897 & -1.099026 \\ \mathrm{H} & 1.611167 & 1.643103 & -1.135026 \\ \mathrm{C} & -2.655833 & -0.010897 & -1.743026 \\ \mathrm{O} & -1.857833 & 0.034103 & -2.672026 \\ \mathrm{O} & -2.364833 & 0.029103 & -0.483026 \\ \mathrm{O} & -0.568833 & 0.169103 & -0.388026 \\ \mathrm{H} & -0.530833 & 0.153103 & -1.375026 \\ \mathrm{H} & -3.745833 & -0.094897 & -1.904026 \\ \mathrm{H} & 0.132167 & 0.360103 & 2.311974 \\ \mathrm{O} & 0.574167 & -1.948897 & 1.339974 \\ \mathrm{H} & -0.270833 & -1.647897 & 0.976974\end{array}$

TS 2c (gas phase) $E=-574.914673 ; v_{i}=-356.4 \mathrm{~cm}^{-1}$

$\begin{array}{rrrr}\text { C } & 1.262814 & -0.241419 & -1.183035 \\ \mathrm{C} & 1.225814 & 2.280581 & -1.205035 \\ \mathrm{C} & 1.278814 & 1.043581 & 1.000965 \\ \mathrm{C} & 0.633814 & 2.185581 & 0.205965 \\ \mathrm{C} & 1.380814 & -0.220419 & 0.180965 \\ \mathrm{C} & 0.992814 & 0.984581 & -1.999035 \\ \mathrm{H} & -0.443186 & 1.999581 & 0.144965 \\ \mathrm{H} & 0.771814 & 3.120581 & 0.754965\end{array}$




$\begin{array}{rrrr}\mathrm{H} & 2.297814 & 1.331581 & 1.287965 \\ \mathrm{H} & 0.792814 & 3.127581 & -1.744035 \\ \mathrm{H} & 2.303814 & 2.473581 & -1.138035 \\ \mathrm{H} & -0.042186 & 0.932581 & -2.358035 \\ \mathrm{H} & 1.619814 & 0.956581 & -2.897035 \\ \mathrm{H} & 1.404814 & -1.181419 & -1.709035 \\ \mathrm{H} & 1.687814 & -1.112419 & 0.716965 \\ \mathrm{O} & 0.616814 & 0.785581 & 2.231965 \\ \mathrm{H} & -0.238186 & 0.389581 & 2.013965 \\ \mathrm{C} & -2.488186 & -2.243419 & 0.302965 \\ \mathrm{O} & -1.591186 & -3.024419 & -0.001035 \\ \mathrm{O} & -2.383186 & -0.961419 & 0.396965 \\ \mathrm{O} & -0.622186 & -0.621419 & -0.039035 \\ \mathrm{H} & -0.526186 & -1.605419 & -0.140035 \\ \mathrm{H} & -3.511186 & -2.593419 & 0.533965\end{array}$

TS 2c $\left.\left(\mathrm{CH}_{2} \mathrm{Cl}_{2}\right)\right)$

$\mathrm{E}=-574.922647$ and (with non electrostatic terms) $-574.922815 ; v_{i}=-383.8 \mathrm{~cm}^{-1}$

$\begin{array}{rrrr}\mathrm{C} & 1.306081 & -0.225465 & -1.171465 \\ \mathrm{C} & 1.290081 & 2.296535 & -1.168465 \\ \mathrm{C} & 1.300081 & 1.032535 & 1.022535 \\ \mathrm{C} & 0.675081 & 2.189535 & 0.232535 \\ \mathrm{C} & 1.408081 & -0.220465 & 0.189535 \\ \mathrm{C} & 1.061081 & 1.010535 & -1.978465 \\ \mathrm{H} & -0.401919 & 2.013535 & 0.151535 \\ \mathrm{H} & 0.813081 & 3.117535 & 0.792535 \\ \mathrm{H} & 2.315081 & 1.311535 & 1.336535 \\ \mathrm{H} & 0.871081 & 3.151535 & -1.702465 \\ \mathrm{H} & 2.368081 & 2.479535 & -1.081465 \\ \mathrm{H} & 0.033081 & 0.970535 & -2.361465 \\ \mathrm{H} & 1.705081 & 0.984535 & -2.864465 \\ \mathrm{H} & 1.447081 & -1.161465 & -1.706465 \\ \mathrm{H} & 1.697081 & -1.124465 & 0.718535 \\ \mathrm{O} & 0.605081 & 0.762535 & 2.240535 \\ \mathrm{H} & -0.241919 & 0.365535 & 1.993535 \\ \mathrm{C} & -2.562919 & -2.236465 & 0.252535 \\ \mathrm{O} & -1.690919 & -3.057465 & -0.003465 \\ \mathrm{O} & -2.408919 & -0.953465 & 0.322535 \\ \mathrm{O} & -0.671919 & -0.633465 & -0.042465 \\ \mathrm{H} & -0.528919 & -1.604465 & -0.143465 \\ \mathrm{H} & -3.608919 & -2.529465 & 0.457535\end{array}$

TS 4 a (gas phase)

$\mathrm{E}=-496.217694 ; v_{\mathrm{i}}=-339.1 \mathrm{~cm}^{-1}$

$\begin{array}{rrrr}\mathrm{C} & -1.629114 & -0.718043 & -0.363043 \\ \mathrm{C} & -1.623114 & 0.815957 & -0.354043 \\ \mathrm{C} & -1.627114 & 0.693957 & 1.229957 \\ \mathrm{C} & -1.760114 & -0.792043 & 1.009957 \\ \mathrm{O} & 2.110886 & -1.266043 & 0.072957 \\ \mathrm{O} & 0.249886 & -1.033043 & 0.201957 \\ \mathrm{C} & 2.583886 & -0.114043 & -0.258043 \\ \mathrm{O} & 1.996886 & 0.927957 & -0.504043 \\ \mathrm{H} & -2.595114 & 1.181957 & -0.701043 \\ \mathrm{H} & -2.466114 & 1.175957 & 1.741957 \\ \mathrm{H} & -1.746114 & -1.442043 & -1.161043 \\ \mathrm{H} & -1.961114 & -1.618043 & 1.681957 \\ \mathrm{H} & 0.240886 & -2.004043 & 0.216957 \\ \mathrm{H} & 3.691886 & -0.160043 & -0.303043\end{array}$




$\begin{array}{rrrr}\mathrm{O} & -0.656114 & 1.535957 & -1.019043 \\ \mathrm{H} & -0.692114 & 0.995957 & 1.701957 \\ \mathrm{H} & 0.248886 & 1.236957 & -0.781043\end{array}$

TS $\left.4 \mathrm{a}\left(\mathrm{CH}_{2} \mathrm{Cl}_{2}\right)\right)$

$E=-496.229419$ and (with non electrostatic terms) $-496.228884 ; v_{i}=-371.3 \mathrm{~cm}^{-1}$

$\begin{array}{lrrr}\mathrm{C} & -1.676771 & -0.707914 & -0.365543 \\ \mathrm{C} & -1.661771 & 0.820086 & -0.342543 \\ \mathrm{C} & -1.655771 & 0.688086 & 1.235457 \\ \mathrm{C} & -1.779771 & -0.797914 & 1.000457 \\ \mathrm{O} & 2.115229 & -1.241914 & 0.130457 \\ \mathrm{O} & 0.313229 & -1.032914 & 0.208457 \\ \mathrm{C} & 2.611229 & -0.117914 & -0.266543 \\ \mathrm{O} & 2.036229 & 0.912086 & -0.583543 \\ \mathrm{H} & -2.623771 & 1.214086 & -0.689543 \\ \mathrm{H} & -2.496771 & 1.158086 & 1.754457 \\ \mathrm{H} & -1.774771 & -1.429914 & -1.168543 \\ \mathrm{H} & -1.969771 & -1.630914 & 1.667457 \\ \mathrm{H} & 0.250229 & -2.005914 & 0.224457 \\ \mathrm{H} & 3.717229 & -0.185914 & -0.293543 \\ \mathrm{O} & -0.667771 & 1.536086 & -1.004543 \\ \mathrm{H} & -0.721771 & 0.994086 & 1.709457 \\ \mathrm{H} & 0.221229 & 1.193086 & -0.778543\end{array}$

TS 4 b (gas phase)

$\mathrm{E}=-535.583624 ; v_{\mathrm{i}}=-325.3 \mathrm{~cm}^{-1}$

$\begin{array}{lrrr}\mathrm{C} & 1.462974 & -0.052936 & -0.871462 \\ \mathrm{C} & 1.463974 & 1.318064 & -0.877462 \\ \mathrm{C} & 1.465974 & 1.875064 & 0.516538 \\ \mathrm{C} & 1.532974 & -0.592936 & 0.544538 \\ \mathrm{C} & 1.166974 & 0.648064 & 1.406538 \\ \mathrm{H} & 2.580974 & -0.876936 & 0.719538 \\ \mathrm{H} & 1.709974 & 0.663064 & 2.351538 \\ \mathrm{H} & 2.459974 & 2.297064 & 0.721538 \\ \mathrm{H} & 0.751974 & 2.694064 & 0.638538 \\ \mathrm{H} & 1.594974 & -0.689936 & -1.736462 \\ \mathrm{H} & 1.537974 & 1.936064 & -1.765462 \\ \mathrm{C} & -2.686026 & -0.678936 & -0.127462 \\ \mathrm{O} & -1.976026 & -1.219936 & 0.704538 \\ \mathrm{O} & -2.368026 & 0.107064 & -1.099462 \\ \mathrm{O} & -0.521026 & 0.379064 & -0.987462 \\ \mathrm{H} & -3.785026 & -0.830936 & -0.115462 \\ \mathrm{H} & -0.582026 & 0.513064 & -1.946462 \\ \mathrm{H} & 0.099974 & 0.599064 & 1.630538 \\ \mathrm{O} & 0.784974 & -1.748936 & 0.787538 \\ \mathrm{H} & -0.169026 & -1.540936 & 0.713538\end{array}$

TS 4b $\left.\left(\mathrm{CH}_{2} \mathrm{Cl}_{2}\right)\right)$

$E=-535.594973$ and (with non electrostatic terms) $-535.595092 ; v_{i}=-364.4 \mathrm{~cm}^{-1}$

$\begin{array}{rrrr}\text { C } & 1.503936 & -0.060551 & -0.844897 \\ \mathrm{C} & 1.519936 & 1.303449 & -0.853897 \\ \mathrm{C} & 1.534936 & 1.867449 & 0.537103 \\ \mathrm{C} & 1.549936 & -0.599551 & 0.569103 \\ \mathrm{C} & 1.220936 & 0.648449 & 1.433103 \\ \mathrm{H} & 2.578936 & -0.934551 & 0.759103 \\ \mathrm{H} & 1.774936 & 0.655449 & 2.372103 \\ \mathrm{H} & 2.535936 & 2.273449 & 0.738103\end{array}$




$\begin{array}{rrrr}\mathrm{H} & 0.835936 & 2.698449 & 0.659103 \\ \mathrm{H} & 1.609936 & -0.698551 & -1.713897 \\ \mathrm{H} & 1.595936 & 1.916449 & -1.745897 \\ \mathrm{C} & -2.733064 & -0.673551 & -0.189897 \\ \mathrm{O} & -2.053064 & -1.313551 & 0.596103 \\ \mathrm{O} & -2.368064 & 0.189449 & -1.080897 \\ \mathrm{O} & -0.578064 & 0.387449 & -0.968897 \\ \mathrm{H} & -3.836064 & -0.782551 & -0.213897 \\ \mathrm{H} & -0.576064 & 0.572449 & -1.924897 \\ \mathrm{H} & 0.154936 & 0.626449 & 1.669103 \\ \mathrm{O} & 0.741936 & -1.731551 & 0.808103 \\ \mathrm{H} & -0.196064 & -1.495551 & 0.662103\end{array}$

TS 4c (gas phase) $E=-574.913978 ; v_{i}=-330.6 \mathrm{~cm}^{-1}$

$\begin{array}{rrrr}\mathrm{C} & 1.344558 & -0.737488 & -1.423686 \\ \mathrm{C} & 1.343558 & 1.787512 & -1.434686 \\ \mathrm{C} & 1.339558 & 0.542512 & 0.769314 \\ \mathrm{C} & 0.726558 & 1.698512 & -0.032686 \\ \mathrm{C} & 1.405558 & -0.726488 & -0.050686 \\ \mathrm{C} & 1.114558 & 0.496512 & -2.238686 \\ \mathrm{H} & -0.353442 & 1.540512 & -0.102686 \\ \mathrm{H} & 0.881558 & 2.627512 & 0.521314 \\ \mathrm{H} & 2.384558 & 0.795512 & 1.005314 \\ \mathrm{H} & 0.929558 & 2.639512 & -1.980686 \\ \mathrm{H} & 2.422558 & 1.969512 & -1.347686 \\ \mathrm{H} & 0.086558 & 0.460512 & -2.622686 \\ \mathrm{H} & 1.761558 & 0.463512 & -3.122686 \\ \mathrm{H} & 1.500558 & -1.673488 & -1.953686 \\ \mathrm{H} & 1.682558 & -1.623488 & 0.493314 \\ \mathrm{O} & 0.721558 & 0.325512 & 2.014314 \\ \mathrm{H} & -0.236442 & 0.173512 & 1.875314 \\ \mathrm{C} & -2.717442 & -0.760488 & 1.011314 \\ \mathrm{O} & -2.057442 & -0.009488 & 1.709314 \\ \mathrm{O} & -2.362442 & -1.478488 & -0.000686 \\ \mathrm{O} & -0.564442 & -1.112488 & -0.359686 \\ \mathrm{H} & -0.497442 & -2.072488 & -0.478686 \\ \mathrm{H} & -3.801442 & -0.904488 & 1.206314 \\ & & & \end{array}$

TS $\left.4 \mathbf{c}\left(\mathrm{CH}_{2} \mathrm{Cl}_{2}\right)\right)$

$E=-574.925134$ and (with non electrostatic terms) $-574.925486 ; v_{i}=-369.0 \mathrm{~cm}^{-1}$

$\begin{array}{lrrr}\mathrm{C} & 1.356779 & -0.721337 & -1.435221 \\ \mathrm{C} & 1.383779 & 1.803663 & -1.456221 \\ \mathrm{C} & 1.361779 & 0.560663 & 0.746779 \\ \mathrm{C} & 0.758779 & 1.719663 & -0.058221 \\ \mathrm{C} & 1.427779 & -0.707337 & -0.070221 \\ \mathrm{C} & 1.147779 & 0.512663 & -2.257221 \\ \mathrm{H} & -0.321221 & 1.563663 & -0.139221 \\ \mathrm{H} & 0.912779 & 2.650663 & 0.493779 \\ \mathrm{H} & 2.399779 & 0.814663 & 1.006779 \\ \mathrm{H} & 0.977779 & 2.657663 & -2.004221 \\ \mathrm{H} & 2.461779 & 1.976663 & -1.363221 \\ \mathrm{H} & 0.124779 & 0.486663 & -2.657221 \\ \mathrm{H} & 1.804779 & 0.467663 & -3.133221 \\ \mathrm{H} & 1.489779 & -1.663337 & -1.961221 \\ \mathrm{H} & 1.681779 & -1.612337 & 0.472779 \\ \mathrm{O} & 0.714779 & 0.347663 & 1.990779 \\ \mathrm{H} & -0.226221 & 0.136663 & 1.826779\end{array}$




$\begin{array}{lllr}\mathrm{C} & -2.743221 & -0.812337 & 1.056779 \\ \mathrm{O} & -2.077221 & -0.122337 & 1.812779 \\ \mathrm{O} & -2.385221 & -1.445337 & -0.013221 \\ \mathrm{O} & -0.641221 & -1.103337 & -0.340221 \\ \mathrm{H} & -0.530221 & -2.051337 & -0.535221 \\ \mathrm{H} & -3.825221 & -0.974337 & 1.233779\end{array}$

TS 6b (gas phase)

$\mathrm{E}=-535.579768 ; v_{\mathrm{i}}=-368.0 \mathrm{~cm}^{-1}$

$\begin{array}{lrrr}\text { C } & 1.204744 & -0.383577 & -0.239654 \\ \mathrm{C} & 1.203744 & 0.983423 & -0.232654 \\ \mathrm{C} & 1.169744 & 1.533423 & 1.160346 \\ \mathrm{C} & 1.251744 & -0.932577 & 1.171346 \\ \mathrm{C} & 0.900744 & 0.288423 & 2.041346 \\ \mathrm{H} & 1.489744 & 0.277423 & 2.958346 \\ \mathrm{H} & 2.138744 & 1.998423 & 1.381346 \\ \mathrm{H} & 0.410744 & 2.311423 & 1.276346 \\ \mathrm{H} & 1.356744 & -1.001577 & -1.115654 \\ \mathrm{H} & 1.292744 & 1.602423 & -1.117654 \\ \mathrm{C} & -2.837256 & -0.042577 & -1.815654 \\ \mathrm{O} & -1.987256 & 0.087423 & -2.692654 \\ \mathrm{O} & -2.620256 & -0.087577 & -0.545654 \\ \mathrm{O} & -0.796256 & 0.083423 & -0.391654 \\ \mathrm{H} & -0.787256 & 0.143423 & -1.382654 \\ \mathrm{H} & -3.911256 & -0.134577 & -2.057654 \\ \mathrm{H} & -0.156256 & 0.249423 & 2.307346 \\ \mathrm{H} & 0.551744 & -1.759577 & 1.316346 \\ \mathrm{O} & 2.588744 & -1.355577 & 1.490346 \\ \mathrm{H} & 2.773744 & -2.187577 & 1.040346\end{array}$

TS $\left.6 \mathbf{b}\left(\mathrm{CH}_{2} \mathrm{Cl}_{2}\right)\right)$

$\mathrm{E}=-574.925134$ and (with non electrostatic terms) $-574.925486 ; v_{\mathrm{i}}=-392.4 \mathrm{~cm}^{-1}$

$\begin{array}{lrrr}\text { C } & 1.242615 & -0.400731 & -0.225077 \\ \mathrm{C} & 1.243615 & 0.961269 & -0.216077 \\ \mathrm{C} & 1.209615 & 1.511269 & 1.176923 \\ \mathrm{C} & 1.275615 & -0.957731 & 1.182923 \\ \mathrm{C} & 0.948615 & 0.266269 & 2.059923 \\ \mathrm{H} & 1.552615 & 0.252269 & 2.967923 \\ \mathrm{H} & 2.175615 & 1.985269 & 1.393923 \\ \mathrm{H} & 0.448615 & 2.287269 & 1.293923 \\ \mathrm{H} & 1.382615 & -1.021731 & -1.102077 \\ \mathrm{H} & 1.339615 & 1.583269 & -1.099077 \\ \mathrm{C} & -2.891385 & 0.016269 & -1.828077 \\ \mathrm{O} & -2.073385 & 0.100269 & -2.736077 \\ \mathrm{O} & -2.629385 & -0.008731 & -0.561077 \\ \mathrm{O} & -0.845385 & 0.102269 & -0.406077 \\ \mathrm{H} & -0.776385 & 0.145269 & -1.389077 \\ \mathrm{H} & -3.978385 & -0.047731 & -2.018077 \\ \mathrm{H} & -0.102385 & 0.232269 & 2.349923 \\ \mathrm{H} & 0.563615 & -1.775731 & 1.324923 \\ \mathrm{O} & 2.605615 & -1.414731 & 1.494923 \\ \mathrm{H} & 2.762615 & -2.252731 & 1.040923\end{array}$


TS 7b (gas phase)

$\mathrm{E}=-535.572842 ; v_{\mathrm{i}}=-363.3 \mathrm{~cm}^{-1}$

$\begin{array}{lrrr}\mathrm{C} & 1.126000 & 0.025910 & -0.957949 \\ \mathrm{C} & 1.086000 & 1.404910 & -0.946949 \\ \mathrm{C} & 1.065000 & 1.943910 & 0.445051 \\ \mathrm{C} & 1.318000 & -0.505090 & 0.454051 \\ \mathrm{C} & 0.909000 & 0.687910 & 1.335051 \\ \mathrm{H} & 1.523000 & 0.721910 & 2.234051 \\ \mathrm{H} & 2.015000 & 2.465910 & 0.628051 \\ \mathrm{H} & 0.274000 & 2.683910 & 0.594051 \\ \mathrm{H} & 1.343000 & -0.576090 & -1.831949 \\ \mathrm{H} & 1.098000 & 2.026910 & -1.834949 \\ \mathrm{C} & -2.779000 & -1.049090 & 0.080051 \\ \mathrm{O} & -2.020000 & -1.223090 & 1.014051 \\ \mathrm{O} & -2.578000 & -0.462090 & -1.055949 \\ \mathrm{O} & -0.773000 & 0.137910 & -1.000949 \\ \mathrm{H} & -0.939000 & 0.351910 & -1.933949 \\ \mathrm{H} & -3.829000 & -1.417090 & 0.117051 \\ \mathrm{H} & -0.135000 & 0.556910 & 1.622051 \\ \mathrm{H} & 0.700000 & -1.382090 & 0.650051 \\ \mathrm{O} & 2.711000 & -0.799090 & 0.655051 \\ \mathrm{H} & 2.880000 & -1.712090 & 0.402051\end{array}$

TS 7b $\left.\left(\mathrm{CH}_{2} \mathrm{Cl}_{2}\right)\right)$

$E=-535.585849$ and (with non electrostatic terms) $-535.585769 ; v_{i}=-371.3 \mathrm{~cm}^{-1}$

$\begin{array}{rrrr}\mathrm{C} & 1.290667 & -0.214833 & -0.949333 \\ \mathrm{C} & 1.196667 & 1.151167 & -0.930333 \\ \mathrm{C} & 1.163667 & 1.684167 & 0.466667 \\ \mathrm{C} & 1.431667 & -0.762833 & 0.457667 \\ \mathrm{C} & 0.991667 & 0.418167 & 1.340667 \\ \mathrm{H} & 1.578667 & 0.448167 & 2.259667 \\ \mathrm{H} & 2.111667 & 2.204167 & 0.663667 \\ \mathrm{H} & 0.369667 & 2.421167 & 0.610667 \\ \mathrm{H} & 1.465667 & -0.815833 & -1.833333 \\ \mathrm{H} & 1.211667 & 1.782167 & -1.812333 \\ \mathrm{C} & -3.039333 & -0.434833 & 0.036667 \\ \mathrm{O} & -2.466333 & -0.413833 & 1.109667 \\ \mathrm{O} & -2.570333 & -0.259833 & -1.159333 \\ \mathrm{O} & -0.757333 & 0.063167 & -1.028333 \\ \mathrm{H} & -0.792333 & 0.165167 & -1.997333 \\ \mathrm{H} & -4.133333 & -0.624833 & -0.031333 \\ \mathrm{H} & -0.061333 & 0.291167 & 1.591667 \\ \mathrm{H} & 0.821667 & -1.655833 & 0.620667 \\ \mathrm{O} & 2.815667 & -1.060833 & 0.717667 \\ \mathrm{H} & 3.044667 & -1.890833 & 0.278667\end{array}$


IRC data (gas phase) at the B3LYP/6-31G(d) level

TS $2 \mathbf{a}$

$E=-496.048967 ; v_{i}=-403.1 \mathrm{~cm}^{-1}$

$\begin{array}{lrrr}\mathrm{C} & -1.559986 & -0.109571 & -0.432457 \\ \mathrm{C} & -1.602986 & 1.418429 & -0.449457 \\ \mathrm{C} & -1.626986 & 1.324429 & 1.134543 \\ \mathrm{C} & -1.628986 & -0.174571 & 0.941543 \\ \mathrm{O} & 2.210014 & -0.319571 & 0.106543 \\ \mathrm{O} & 0.372014 & -0.246571 & 0.197543 \\ \mathrm{C} & 2.442014 & -1.569571 & -0.125457 \\ \mathrm{O} & 1.589014 & -2.455571 & -0.241457 \\ \mathrm{H} & -2.541986 & 1.813429 & -0.852457 \\ \mathrm{H} & -2.520986 & 1.736429 & 1.617543 \\ \mathrm{H} & -1.610986 & -0.855571 & -1.219457 \\ \mathrm{H} & -1.743986 & -1.004571 & 1.632543 \\ \mathrm{H} & 0.417014 & -1.238571 & 0.032543 \\ \mathrm{H} & 3.520014 & -1.794571 & -0.213457 \\ \mathrm{O} & -0.567986 & 2.103429 & -1.083457 \\ \mathrm{H} & -0.733986 & 1.727429 & 1.619543 \\ \mathrm{H} & 0.252014 & 1.627429 & -0.862457\end{array}$

\section{IRC-9a}

$\begin{array}{rrrr}\mathrm{C} & -1.440886 & 0.018057 & -0.407200 \\ \mathrm{C} & -1.440886 & 1.558057 & -0.407200 \\ \mathrm{C} & -1.440886 & 1.469057 & 1.180800 \\ \mathrm{C} & -1.504886 & -0.033943 & 1.016800 \\ \mathrm{O} & 2.260114 & -0.550943 & 0.019800 \\ \mathrm{O} & 0.080114 & -0.268943 & 0.194800 \\ \mathrm{C} & 2.288114 & -1.779943 & -0.197200 \\ \mathrm{O} & 1.270114 & -2.542943 & -0.271200 \\ \mathrm{H} & -2.371886 & 1.988057 & -0.790200 \\ \mathrm{H} & -2.316886 & 1.915057 & 1.665800 \\ \mathrm{H} & -1.698886 & -0.702943 & -1.176200 \\ \mathrm{H} & -1.721886 & -0.847943 & 1.698800 \\ \mathrm{H} & 0.381114 & -1.350943 & 0.018800 \\ \mathrm{H} & 3.277114 & -2.261943 & -0.334200 \\ \mathrm{O} & -0.384886 & 2.163057 & -1.069200 \\ \mathrm{H} & -0.530886 & 1.828057 & 1.666800 \\ \mathrm{H} & 0.415114 & 1.643057 & -0.859200\end{array}$

TS $4 \mathbf{a}$

$E=-496.047509 ; v_{i}=-371.1 \mathrm{~cm}^{-1}$

$\begin{array}{lrrrr}\mathrm{C} & -1.584443 & -0.756457 & -0.410457 & \\ \mathrm{C} & -1.611443 & 0.780543 & -0.408457 & \\ \mathrm{C} & -1.658443 & 0.663543 & 1.177543 & \\ \mathrm{C} & -1.754443 & -0.826457 & 0.962543 & \\ \mathrm{O} & 2.102557 & -1.240457 & 0.089543 & \\ \mathrm{O} & 0.239557 & -1.016457 & 0.195543 & \\ \mathrm{C} & 2.572557 & -0.066457 & -0.166457 & \\ \mathrm{O} & 1.987557 & 0.990543 & -0.381457 & \\ \mathrm{H} & -2.583443 & 1.125543 & -0.784457 & \\ \mathrm{H} & -2.521443 & 1.132543 & 1.666543 & \\ \mathrm{H} & -1.673443 & -1.486457 & -1.208457 & \\ \mathrm{H} & -1.955443 & -1.654457 & 1.636543 & \\ \mathrm{H} & 0.248557 & - & 1.993457 & 0.205543\end{array}$




$\begin{array}{rrrr}\mathrm{H} & 3.683557 & -0.093457 & -0.175457 \\ \mathrm{O} & -0.642443 & 1.514543 & -1.050457 \\ \mathrm{H} & -0.739443 & 0.991543 & 1.671543 \\ \mathrm{H} & 0.260557 & 1.224543 & -0.765457\end{array}$

\section{IRC-10a}

$\begin{array}{lrrr}\mathrm{C} & -1.522071 & -0.648571 & -0.333086 \\ \mathrm{C} & -1.522071 & 0.905429 & -0.333086 \\ \mathrm{C} & -1.522071 & 0.811429 & 1.263914 \\ \mathrm{C} & -1.701071 & -0.674571 & 1.098914 \\ \mathrm{O} & 2.040929 & -1.454571 & -0.051086 \\ \mathrm{O} & -0.106071 & -0.945571 & 0.226914 \\ \mathrm{C} & 2.545929 & -0.316571 & -0.308086 \\ \mathrm{O} & 2.012929 & 0.794429 & -0.509086 \\ \mathrm{H} & -2.502071 & 1.264429 & -0.672086 \\ \mathrm{H} & -2.350071 & 1.325429 & 1.765914 \\ \mathrm{H} & -1.832071 & -1.344571 & -1.107086 \\ \mathrm{H} & -1.926071 & -1.488571 & 1.779914 \\ \mathrm{H} & 0.283929 & -1.856571 & 0.109914 \\ \mathrm{H} & 3.659929 & -0.338571 & -0.350086 \\ \mathrm{O} & -0.547071 & 1.565429 & -1.018086 \\ \mathrm{H} & -0.578071 & 1.089429 & 1.740914 \\ \mathrm{H} & 0.366929 & 1.208429 & -0.788086\end{array}$

TS 7b

$E=-535.388985 ; v_{i}=-395.2 \mathrm{~cm}^{-1}$

$\begin{array}{rrrr}\mathrm{C} & 1.049859 & -0.175115 & -0.969141 \\ \mathrm{C} & 1.242859 & 1.194885 & -1.024141 \\ \mathrm{C} & 1.350859 & 1.789885 & 0.344859 \\ \mathrm{C} & 1.211859 & -0.667115 & 0.463859 \\ \mathrm{C} & 1.000859 & 0.613885 & 1.290859 \\ \mathrm{H} & 1.617859 & 0.590885 & 2.191859 \\ \mathrm{H} & 2.383859 & 2.143885 & 0.489859 \\ \mathrm{H} & 0.701859 & 2.663885 & 0.477859 \\ \mathrm{H} & 1.157859 & -0.844115 & -1.817141 \\ \mathrm{H} & 1.333859 & 1.769885 & -1.942141 \\ \mathrm{C} & -2.855141 & -0.665115 & 0.161859 \\ \mathrm{O} & -2.101141 & -0.903115 & 1.093859 \\ \mathrm{O} & -2.615141 & -0.142115 & -1.000141 \\ \mathrm{O} & -0.760141 & 0.230885 & -0.983141 \\ \mathrm{H} & -0.910141 & 0.429885 & -1.927141 \\ \mathrm{H} & -3.942141 & -0.907115 & 0.234859 \\ \mathrm{H} & -0.052141 & 0.660885 & 1.577859 \\ \mathrm{H} & 0.476859 & -1.435115 & 0.719859 \\ \mathrm{O} & 2.554859 & -1.132115 & 0.645859 \\ \mathrm{H} & 2.597859 & -2.049115 & 0.333859\end{array}$

IRC $-11 b$

$\begin{array}{lrrr}\text { C } & 0.000000 & 0.000000 & 0.000000 \\ \mathrm{C} & 0.000000 & 0.000000 & 1.448273 \\ \mathrm{C} & 1.391525 & 0.000000 & 1.979042 \\ \mathrm{C} & 1.426482 & 0.335337 & -0.465118 \\ \mathrm{C} & 2.288015 & -0.135814 & 0.721189 \\ \mathrm{H} & 3.201767 & 0.458495 & 0.786618 \\ \mathrm{H} & 1.545991 & 0.963640 & 2.493791 \\ \mathrm{H} & 1.552941 & -0.781711 & 2.729852 \\ \mathrm{H} & -0.849422 & 0.365487 & -0.574735 \\ \mathrm{H} & -0.900143 & -0.033275 & 2.053278\end{array}$




$\begin{array}{rrrr}\mathrm{C} & 0.973725 & -3.631751 & -1.257144 \\ \mathrm{O} & 1.914170 & -2.834005 & -1.372178 \\ \mathrm{O} & -0.170844 & -3.521835 & -0.698413 \\ \mathrm{O} & -0.012592 & -1.489925 & 0.203229 \\ \mathrm{H} & -0.836058 & -2.009359 & -0.007837 \\ \mathrm{H} & 1.108094 & -4.648376 & -1.708433 \\ \mathrm{H} & 2.545135 & -1.183469 & 0.549935 \\ \mathrm{H} & 1.669316 & -0.226867 & -1.371388 \\ \mathrm{O} & 1.582479 & 1.744370 & -0.627089 \\ \mathrm{H} & 1.278324 & 1.981701 & -1.516524\end{array}$

\section{$\operatorname{IRC}-11 b^{\prime}$}

$\begin{array}{lrrr}\mathrm{C} & 0.968372 & -0.000154 & -0.912962 \\ \mathrm{C} & 0.968372 & 1.458846 & -0.912962 \\ \mathrm{C} & 0.968372 & 1.989846 & 0.482038 \\ \mathrm{C} & 1.275372 & -0.459154 & 0.517038 \\ \mathrm{C} & 0.796372 & 0.731846 & 1.371038 \\ \mathrm{H} & 1.371372 & 0.788846 & 2.298038 \\ \mathrm{H} & 1.941372 & 2.481846 & 0.645038 \\ \mathrm{H} & 0.199372 & 2.754846 & 0.633038 \\ \mathrm{H} & 1.360372 & -0.567154 & -1.757962 \\ \mathrm{H} & 0.973372 & 2.062846 & -1.813962 \\ \mathrm{C} & -2.689628 & -1.202154 & -0.036962 \\ \mathrm{O} & -1.911628 & -1.326154 & 0.909038 \\ \mathrm{O} & -2.541628 & -0.643154 & -1.199962 \\ \mathrm{O} & -0.454628 & 0.286846 & -0.955962 \\ \mathrm{H} & -1.373628 & -0.195154 & -1.501962 \\ \mathrm{H} & -3.716628 & -1.616154 & 0.064038 \\ \mathrm{H} & -0.257628 & 0.575846 & 1.604038 \\ \mathrm{H} & 0.700372 & -1.360154 & 0.751038 \\ \mathrm{O} & 2.678372 & -0.632154 & 0.701038 \\ \mathrm{H} & 2.914372 & -1.523154 & 0.402038\end{array}$

HCOOH-epoxide complex

$\begin{array}{lrrr}\text { C } & 1.107923 & -0.152077 & -0.739410 \\ \text { C } & 1.107923 & 1.313923 & -0.739410 \\ \text { C } & 1.107923 & 1.841923 & 0.677590 \\ \text { C } & 1.133923 & -0.631077 & 0.696590 \\ \text { C } & 0.676923 & 0.603923 & 1.503590 \\ \text { H } & 1.117923 & 0.581923 & 2.502590 \\ \text { H } & 2.119923 & 2.176923 & 0.937590 \\ \text { H } & 0.430923 & 2.693923 & 0.800590 \\ \text { H } & 1.535923 & -0.749077 & -1.544410 \\ \text { H } & 1.426923 & 1.901923 & -1.594410 \\ \mathrm{C} & -2.815077 & -0.887077 & -0.446410 \\ \mathrm{O} & -2.154077 & -1.097077 & 0.549590 \\ \mathrm{O} & -2.430077 & -0.334077 & -1.593410 \\ \mathrm{O} & -0.120077 & 0.494923 & -1.012410 \\ \mathrm{H} & -1.491077 & -0.032077 & -1.514410 \\ \mathrm{H} & -3.882077 & -1.158077 & -0.519410 \\ \mathrm{H} & -0.409077 & 0.572923 & 1.596590 \\ \mathrm{H} & 0.446923 & -1.471077 & 0.851590 \\ \mathrm{O} & 2.470923 & -0.963077 & 1.059590 \\ \mathrm{H} & 2.652923 & -1.860077 & 0.741590\end{array}$




\section{Absolute value of thermodynamic parameters $(E, H$ and $G$ in hartree and $S$ in e.u.) of the starting reactants.}

(at 298.15 K; these data were taken from Gaussian output and no correction has been added. For contributions, as required by change in standard state, introduced in the evaluation of activation parameters see Computational methods and ref. 17 . Solvent data include all non electrostatic terms).

s-cis $\mathrm{HCOOOH}: \mathrm{E}_{\text {gas }}\left(\mathrm{C}_{6} \mathrm{H}_{6}\right) \quad\left[\mathrm{CH}_{2} \mathrm{Cl}_{2}\right]=-264.879665 \quad(-264.880441) \quad$ [264.882978]; $\mathrm{H}_{\text {gas }}\left(\mathrm{C}_{6} \mathrm{H}_{6}\right) \quad\left[\mathrm{CH}_{2} \mathrm{Cl}_{2}\right] \quad=-264.837440 \quad(-264.838366)$ [264.841062], $\mathrm{S}_{\text {gas }}\left(\mathrm{C}_{6} \mathrm{H}_{6}\right) \quad\left[\mathrm{CH}_{2} \mathrm{Cl}_{2}\right]=65.33(65.51) \quad[65.71], \quad \mathrm{G}_{\text {gas }}$ $\left(\mathrm{C}_{6} \mathrm{H}_{6}\right) \quad\left[\mathrm{CH}_{2} \mathrm{Cl}_{2}\right]=-264.868481 \quad(-264.869491) \quad[-264.872284]$ at the B3LYP/6-31G(d) level. $\mathrm{E}_{\text {gas }} \quad\left(\mathrm{C}_{6} \mathrm{H}_{6}\right) \quad\left[\mathrm{CH}_{2} \mathrm{Cl} l_{2}\right]=\quad-264.972914 \quad(-$ $264.974071)[-264.976919] ; \quad \mathrm{H}_{\mathrm{gas}} \quad\left(\mathrm{C}_{6} \mathrm{H}_{6}\right) \quad\left[\mathrm{CH}_{2} \mathrm{Cl}_{2}\right] \quad-264.972914 \quad(-$ 264.932253), [-264.935333], $\mathrm{S}_{\text {gas }} \quad\left(\mathrm{C}_{6} \mathrm{H}_{6}\right) \quad\left[\mathrm{CH}_{2} \mathrm{Cl}_{2}\right] \quad 65.45 \quad(65.64)$ $[65.93]$ and $\mathrm{G}_{\text {gas }}\left(\mathrm{C}_{6} \mathrm{H}_{6}\right) \quad\left[\mathrm{CH}_{2} \mathrm{Cl}_{2}\right]=-264.962057 \quad(-264.963441) \quad[-$ 264.966658] at the B3LYP/6-311+G(d,p) level.

2-cyclobutenol: $E_{\text {gas }} \quad\left(\mathrm{C}_{6} \mathrm{H}_{6}\right) \quad\left[\begin{array}{lllll}\left.\mathrm{CH}_{2} \mathrm{Cl}_{2}\right] & -231.183256 & (-231.183886) & \text { [- }\end{array}\right.$ 231.187718]; $\mathrm{H}_{\text {gas }} \quad\left(\mathrm{C}_{6} \mathrm{H}_{6}\right) \quad\left[\mathrm{CH}_{2} \mathrm{Cl}_{2}\right]=-231.085995 \quad(-231.086781) \quad[-$ $231.090779 ; \mathrm{S}_{\text {gas }}\left(\mathrm{C}_{6} \mathrm{H}_{6}\right) \quad\left[\mathrm{CH}_{2} \mathrm{Cl}_{2}\right]=69.73(69.74)[69.75], \mathrm{G}_{\text {gas }}\left(\mathrm{C}_{6} \mathrm{H}_{6}\right)$ $\left[\mathrm{CH}_{2} \mathrm{Cl}_{2}\right]-231.119127(-231.119914)$ [-231.123921] the B3LYP/6-31G(d) level. $\mathrm{E}_{\text {gas }}\left(\mathrm{C}_{6} \mathrm{H}_{6}\right) \quad\left[\mathrm{CH}_{2} \mathrm{Cl}_{2}\right]-231.260864(-231.185019)$ [-231.266122], $\begin{array}{lllllll}\mathrm{H}_{\text {gas }} & \left(\mathrm{C}_{6} \mathrm{H}_{6}\right) & {\left[\mathrm{CH}_{2} \mathrm{Cl}_{2}\right]} & -231.164202 & (-231.165455) & {[-231.1169872],} & \mathrm{S}_{\text {gas }}\end{array}$ $\left(\mathrm{C}_{6} \mathrm{H}_{6}\right) \quad\left[\mathrm{CH}_{2} \mathrm{Cl}_{2}\right]=69.86(69.74) \quad[69.75]$ and $\mathrm{G}_{\text {gas }}\left(\mathrm{C}_{6} \mathrm{H}_{6}\right) \quad\left[\mathrm{CH}_{2} \mathrm{Cl}_{2}\right]=-$ $231.197397(-231.198678) \quad[-231.202103]$ at the B3LYP/6-311+G $(\mathrm{d}, \mathrm{p})$ level.

2-cyclopentenol: $\quad E_{\text {gas }} \quad\left(\mathrm{C}_{6} \mathrm{H}_{6}\right) \quad\left[\mathrm{CH}_{2} \mathrm{Cl}_{2}\right]-270.536591 \quad(-270.538086) \quad[-$ $270.542008] ; \quad \mathrm{H}_{\text {gas }} \quad\left(\mathrm{C}_{6} \mathrm{H}_{6}\right) \quad\left[\begin{array}{llll}\left.\mathrm{CH}_{2} \mathrm{Cl}_{2}\right] & -270.408175 & (-270.409824) \quad[-\end{array}\right.$ 270.4139008], $\mathrm{S}_{\text {gas }}\left(\mathrm{C}_{6} \mathrm{H}_{6}\right) \quad\left[\mathrm{CH}_{2} \mathrm{Cl}_{2}\right]=75.46$ (75.55) [75.71], Ggas $\left(\begin{array}{lllll}\left(\mathrm{C}_{6} \mathrm{H}_{6}\right) & {\left[\mathrm{CH}_{2} \mathrm{Cl}_{2}\right]} & -270.444029 & (-270.445719) \quad[-270.449880\end{array}\right]$ the B3LYP/6-31G (d) level. $E_{\text {gas }}\left(\mathrm{C}_{6} \mathrm{H}_{6}\right) \quad\left[\mathrm{CH}_{2} \mathrm{Cl}_{2}\right]-270.625121 \quad(-270.627062)$ $[-270.631369], \mathrm{H}_{\text {gas }} \quad\left(\mathrm{C}_{6} \mathrm{H}_{6}\right) \quad\left[\mathrm{CH}_{2} \mathrm{Cl}_{2}\right]=-270.497535 \quad(-270.499671) \quad[-$ 270.504193], $\mathrm{S}_{\text {gas }}\left(\mathrm{C}_{6} \mathrm{H}_{6}\right) \quad\left[\mathrm{CH}_{2} \mathrm{Cl}_{2}\right]=75.50 \quad(75.61) \quad[75.80]$ and $\mathrm{G}_{\text {gas }}$ $\left(\mathrm{C}_{6} \mathrm{H}_{6}\right) \quad\left[\mathrm{CH}_{2} \mathrm{Cl}_{2}\right]=-270.533407(-270.535593) \quad[-270.540206]$ at the B3LYP / 6-311+G (d,p) level 
2-cyclohexenol: $\quad E_{\text {gas }} \quad\left(\mathrm{C}_{6} \mathrm{H}_{6}\right) \quad\left[\mathrm{CH}_{2} \mathrm{Cl}_{2}\right]-309.857402 \quad(-309.859849) \quad$ [$309.863438] ; \mathrm{H}_{\text {gas }}\left(\mathrm{C}_{6} \mathrm{H}_{6}\right) \quad\left[\mathrm{CH}_{2} \mathrm{Cl}_{2}\right]=-309.698504 \quad(-309.701092) \quad[-$ 309.704828], $\mathrm{S}_{\text {gas }}\left(\mathrm{C}_{6} \mathrm{H}_{6}\right) \quad\left[\mathrm{CH}_{2} \mathrm{Cl}_{2}\right]=79.31 \quad(79.35) \quad[79.40]$ and $\mathrm{G}_{\text {gas }}$ $\left(\mathrm{C}_{6} \mathrm{H}_{6}\right) \quad\left[\mathrm{CH}_{2} \mathrm{Cl}_{2}\right]=-309.736186(-309.738794) \quad[-309.742551]$ at the B3LYP/6-31G(d) level. Egas $\left(\mathrm{C}_{6} \mathrm{H}_{6}\right) \quad\left[\mathrm{CH}_{2} \mathrm{Cl}_{2}\right]-309.955675$ (-309.958543) $[-309.962477]$, and $\mathrm{H}_{\text {gas }}\left(\mathrm{C}_{6} \mathrm{H}_{6}\right) \quad\left[\mathrm{CH}_{2} \mathrm{Cl}_{2}\right]=-309.797864 \quad(-309.800909) \quad$ [309.805041], $\mathrm{S}_{\text {gas }}\left(\mathrm{C}_{6} \mathrm{H}_{6}\right) \quad\left[\mathrm{CH}_{2} \mathrm{Cl}_{2}\right]=79.58 \quad(79.65) \quad[79.74]$ and $\mathrm{G}_{\text {gas }}$ $\left(\mathrm{C}_{6} \mathrm{H}_{6}\right) \quad\left[\mathrm{CH}_{2} \mathrm{Cl}_{2}\right]=-309.835677 \quad(-309.838754) \quad[-309.842929]$ at the B3LYP / 6-311+G (d,p) level. 


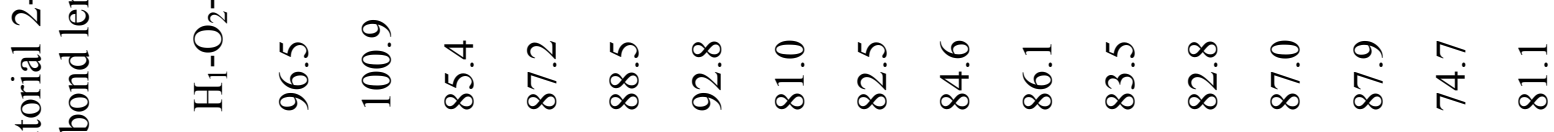

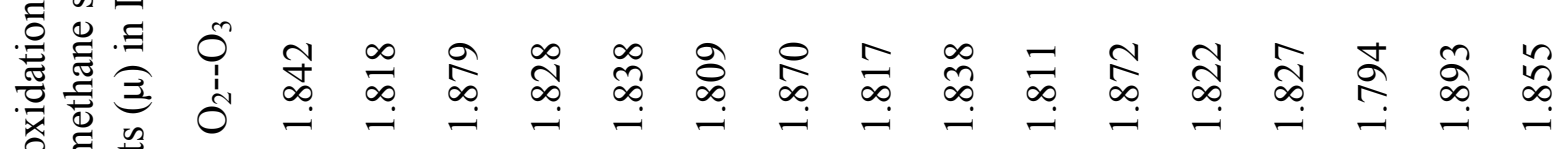
응 율

棺 趈 $\frac{0}{0}$

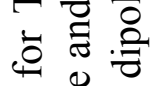

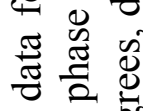

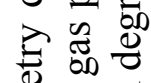

范.$\Xi$

$\stackrel{0}{0} \frac{0}{0}$

的完

응

ज苛

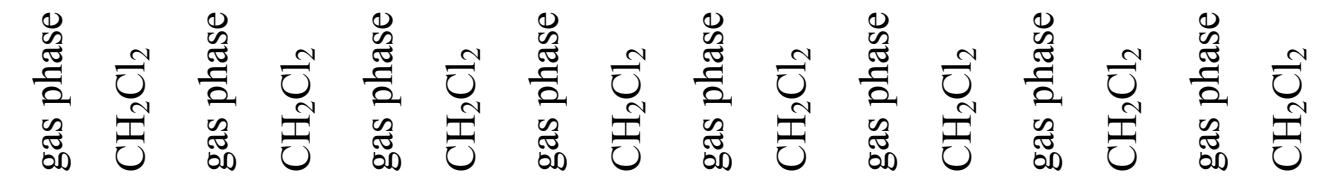
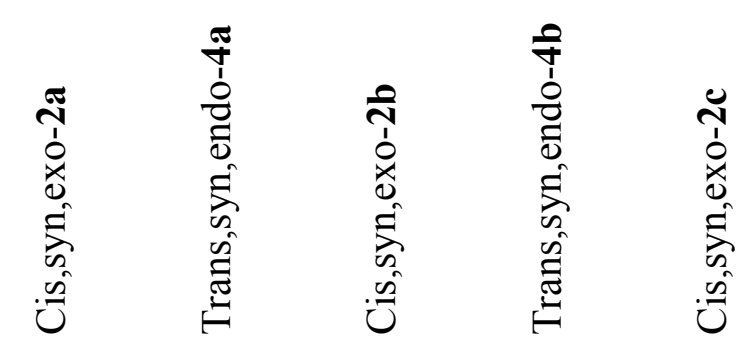

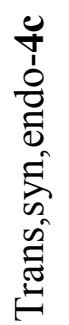




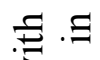

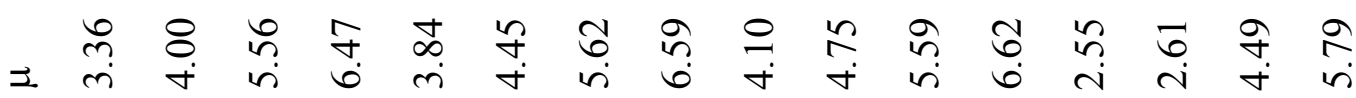

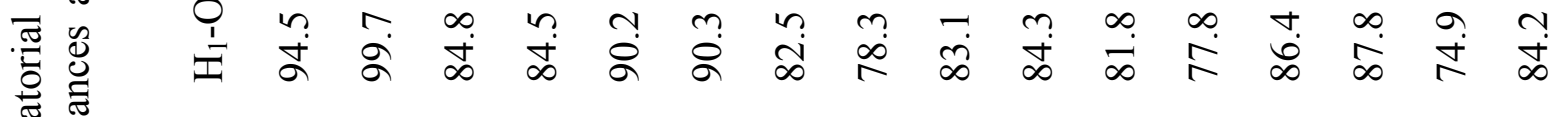

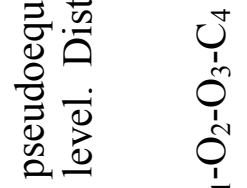

छ)

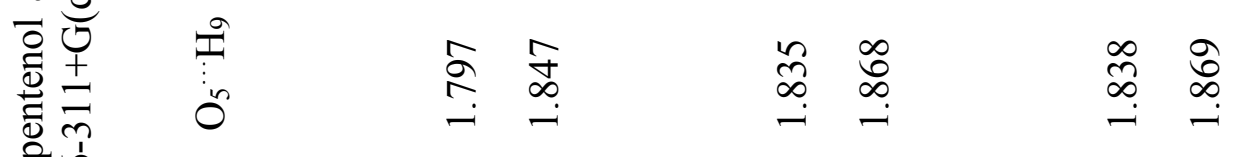

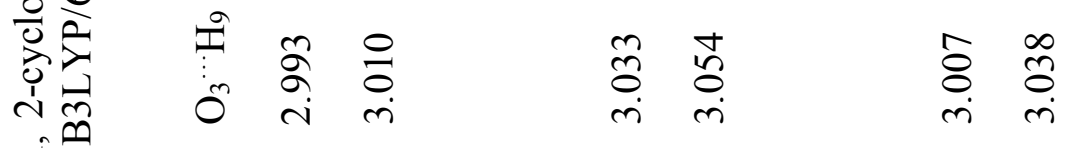

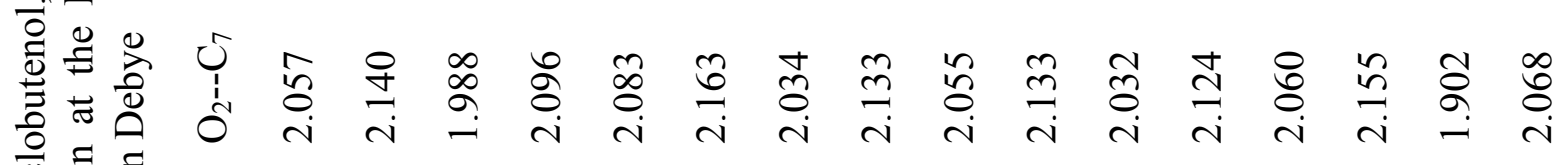
응 ․․

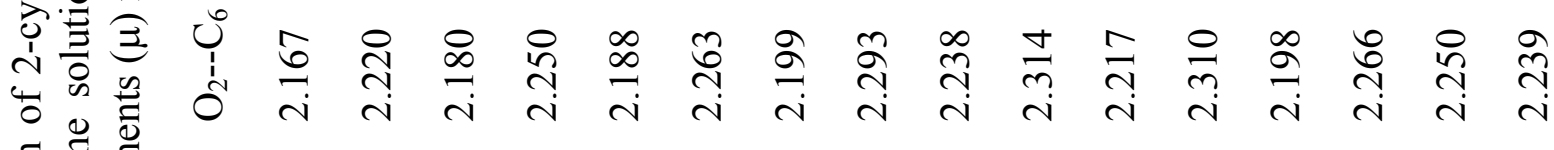

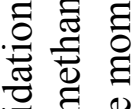

రิ)

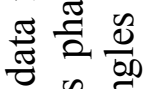

入

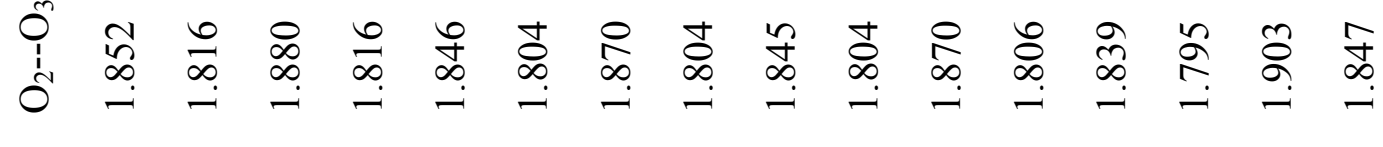

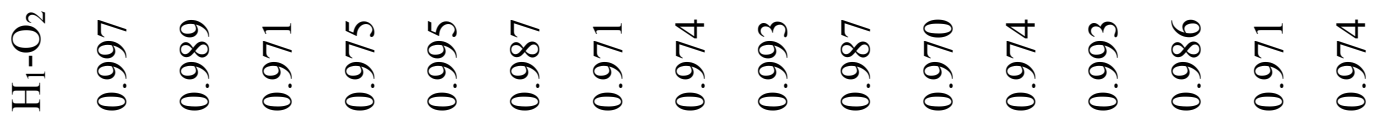

突

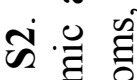

을

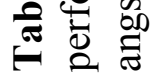

W
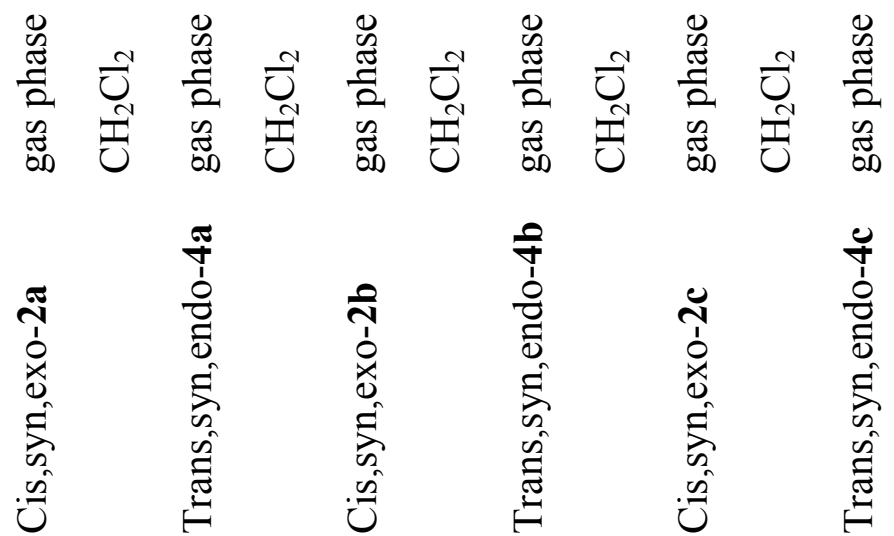

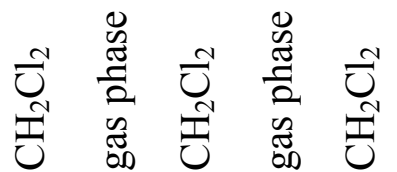

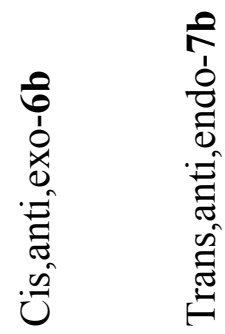




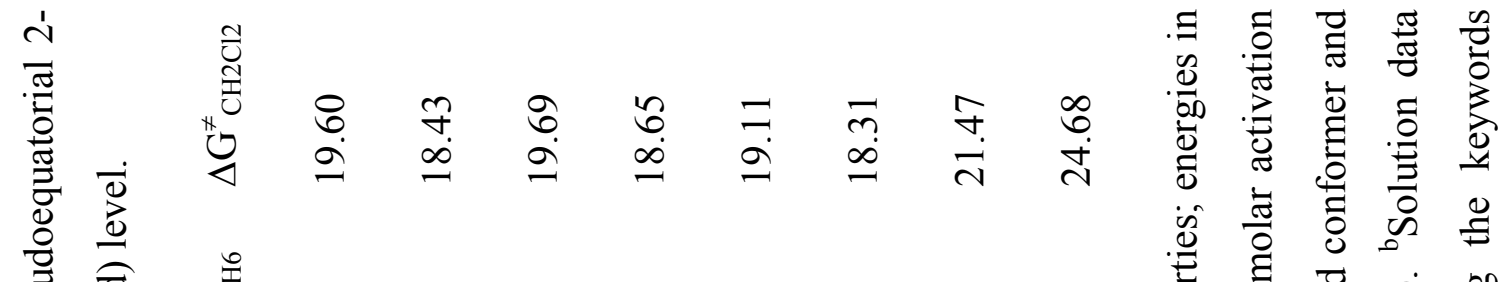

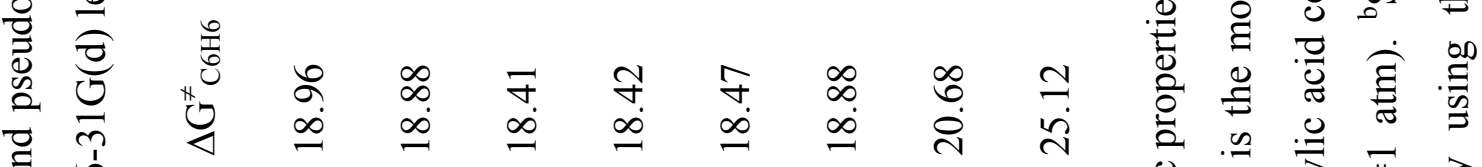

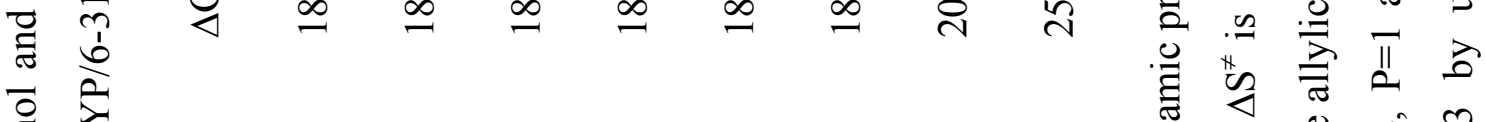

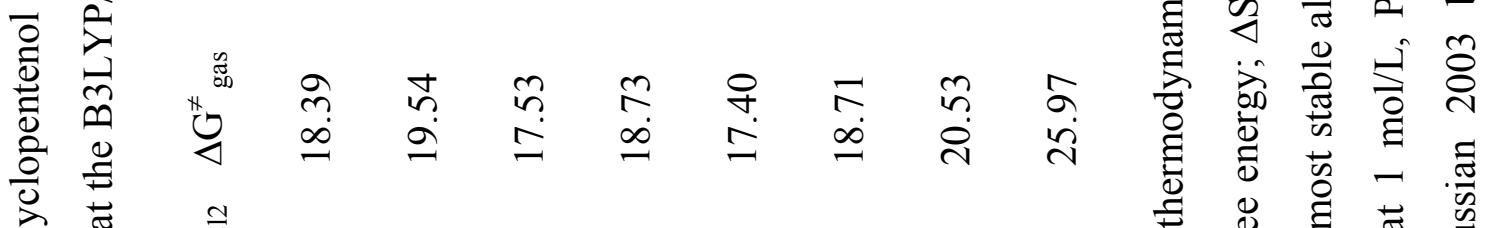

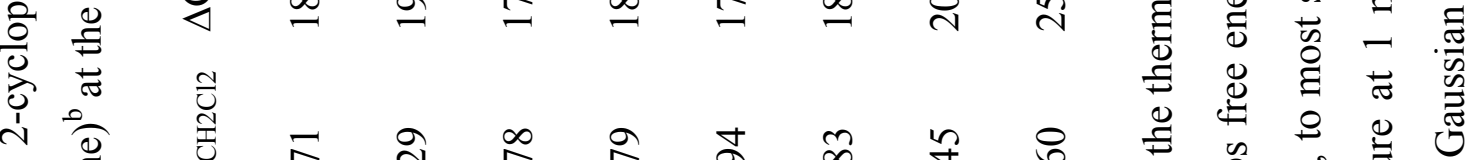

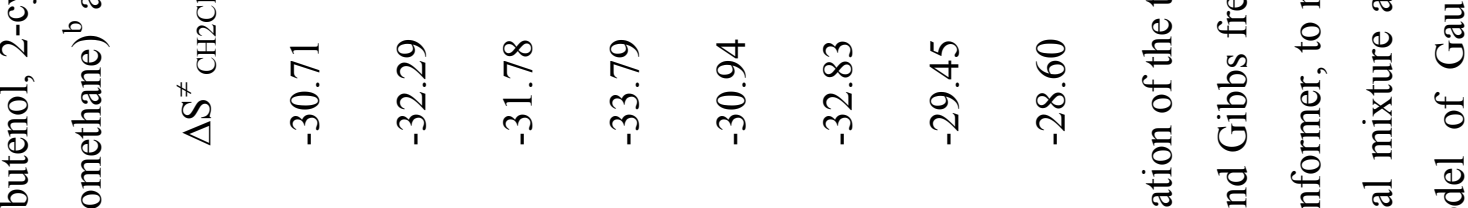

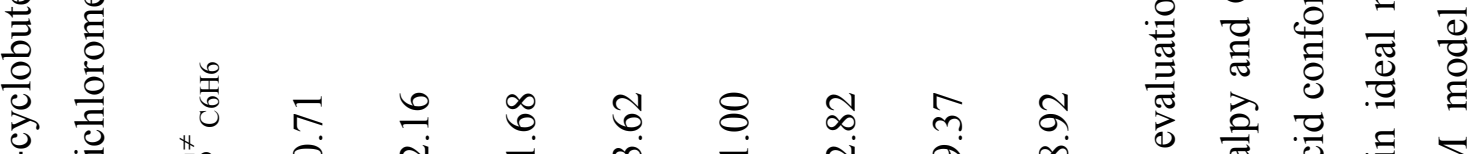

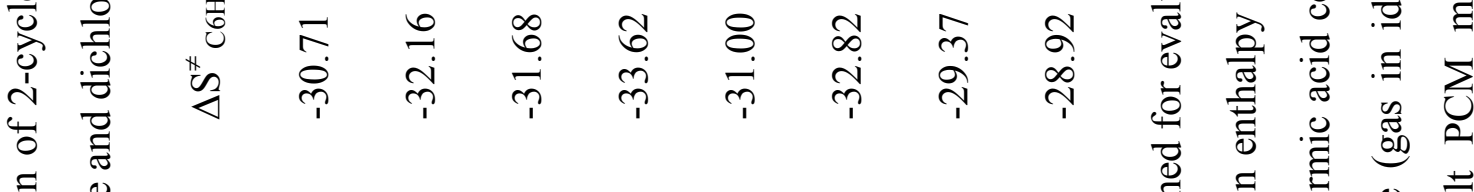

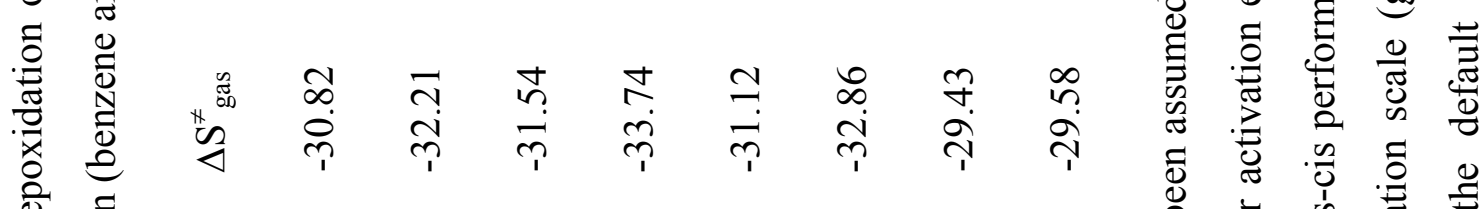

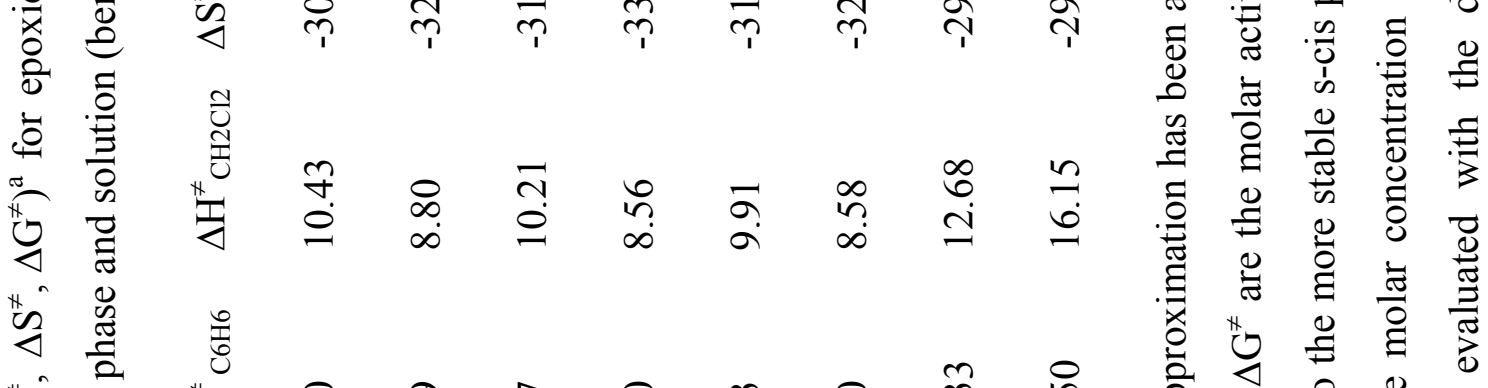

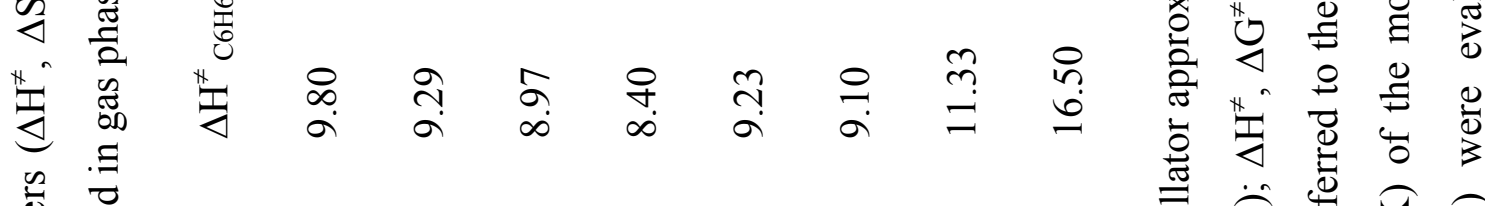

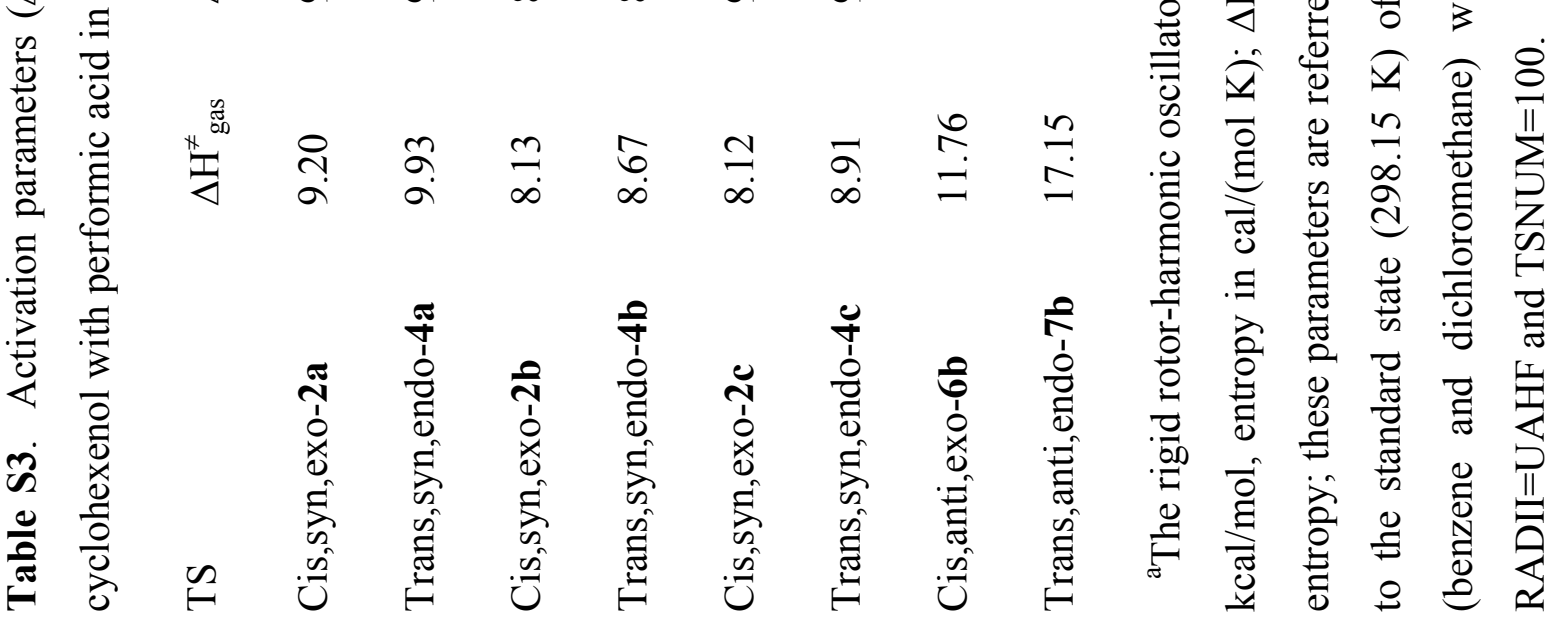

